\title{
HUtrista
}

\section{Efeito da prática regular de atividade física na qualidade de vida relacionada à saúde de hipertensos resistentes}

\section{Effect of regular physical activity on health-related quality of life of resistant}

hypertensive patients

\section{Natália Portela Pereira \\ Gabriela Galdino Matias}

Isabelle Magalhães Guedes Freitas ${ }^{1,2}$ Leonardo Barbosa de Almeida Pedro Augusto de Carvalho Mira ${ }^{1,3}$

Rogério Baumgratz de Paula ${ }^{4}$ Daniel Godoy Martinez ${ }^{1}$ Mateus Camaroti Laterza

${ }^{1}$ Unidade de Investigação Cardiovascular e Fisiologia do Exercício, Hospital Universitário e Faculdade de Educação Física e Desporto Universidade Federal de Fora, Brasil.

${ }^{2}$ Faculdade de Ciências Médicas e da Saúde de Juiz de Fora - SUPREMA, Brasil.

'Laboratório de Ciências do Exercício, Departamento de Fisiologia Farmacologia, Universidade Federal Fluminense, Brasil.

${ }^{4}$ Núcleo Interdisciplinar de Estudos, Pesquisas e Tratamento em Nefrologia NIEPEN, Universidade Federal de Juiz de Fora, Brasil.

\section{Mateus Laterza}

Campus Universitário, s/n, Martelos, Juiz de Fora, Minas Gerais CEP: $36036-900$

A mateuslaterza@hotmail.com

\section{RESUMO}

Introdução: Hipertensos resistentes apresentam pior qualidade de vida relacionada à saúde em relação aos pacientes com hipertensão arterial sistêmica. A prática regular de exercícios físicos melhora essa qualidade de vida de pacientes com hipertensão arterial sistêmica. Assim, é possível que a atividade física melhore a qualidade de vida relacionada à saúde dos hipertensos resistentes. Objetivo: Avaliar os efeitos da prática regular de atividade física na qualidade de vida relacionada à saúde de pacientes com hipertensão arterial resistente. Material e Métodos: Foram avaliados 38 pacientes, de ambos os gêneros, diagnosticados com hipertensão arterial resistente, divididos nos grupos ativo ( $n=19,64 \pm 7$ anos) e sedentário $(n=19,56 \pm 10$ anos). Foi considerado fisicamente ativo o paciente que praticava exercício físico por pelo menos três vezes por semana, com duração mínima de duas horas semanais, por período superior a quatro meses. Foi utilizado o teste $t$ de Student para amostras independentes, com nível de significância de $p<0,05$. Resultados: A qualidade de vida relacionada à saúde foi significativamente maior no grupo Ativo em relação ao grupo Sedentário para os domínios capacidade funcional ( $69 \pm 25$ vs. $44 \pm 22$ pontos, $p<0,01)$, dor $(66 \pm 23$ vs. $49 \pm 22$ pontos, $p=0,03$ ), estado geral de saúde ( $61 \pm 16$ vs. $52 \pm 9$ pontos, $p=0,03)$, vitalidade $(69 \pm 20$ vs. $43 \pm 22$ pontos, $p<0,01)$, limitação por aspectos físicos ( $75 \pm 38$ vs. $40 \pm 34$ pontos, $p<0,01)$ e saúde mental $(76 \pm 24$ vs. $53 \pm 26$ pontos, $p=0,01)$, respectivamente. Os grupos Ativo e Sedentário foram semelhantes para os domínios aspectos sociais ( $74 \pm 24$ vs. $68 \pm 28$ pontos, $p=0,54)$ e limitação por aspectos emocionais ( $63 \pm 38$ vs. $40 \pm 36$ pontos, $p=0,07)$, respectivamente. Conclusão: A prática regular de atividade física parece melhorar a qualidade de vida relacionada à saúde de hipertensos resistentes.

Palavras-chaves: Hipertensão; Doença Crônica; Qualidade de Vida; Atividade Física.

\section{ABSTRACT}

Introduction: Resistant hypertensive patients have worse health-related quality of life than patients with systemic arterial hypertension. Regular exercise improves this quality of life of patients with systemic arterial hypertension. Thus, it is possible that physical activity increases the health-related quality of life of resistant hypertensive patients. Objective: To test the hypothesis that regular physical activity improves the health-related quality of life of patients with resistant hypertension. Material and methods: Were evaluated 38 patients, male and famale, diagnosed with resistant hypertension, divided into Active ( $n=19,64 \pm 7$ years old) and Sedentary ( $n=19,56 \pm 10$ years old) groups. The patient who was physically active for at least three times a week, with a minimum duration of two hours per week for more than four months, was considered physically active. And, considered sedentary, the patient who had not practiced exercise regularly for at least six months. Student's t test for independent samples was used and considered significant $p<0.05$. Results: Health-related quality of life was significantly higher in the Active group compared to the Sedentary group for the domains functional capacity ( $69 \pm 25$ vs. $44 \pm 22$ points, $p<0.01)$, pain ( $66 \pm 23$ vs. $49 \pm 22$ points, $p=0.03$ ), general health ( $61 \pm 16$ vs. $52 \pm 9$ points, $p=0.03)$, vitality $(69 \pm 20$ vs. $43 \pm 22$ points, $p<0.01)$, limitation by physical aspects ( $75 \pm 38$ vs. $40 \pm 34$ points, $p<0.01)$ and mental health $(76 \pm 24$ vs. $53 \pm 26$ points, $p=0.01)$, respectively. And, the Active and Sedentary groups were similar for the domains social aspects ( $74 \pm 24$ vs. $68 \pm 28$ points, $p=0.54)$ and limitation by emotional aspects ( $63 \pm 38$ vs. $40 \pm 36$ points, $p=0.07)$, respectively. Conclusion: Regular physical activity improves the health-related quality of life of patients with resistant hypertension.

Key-words: Hypertension; Chronic Disease; Quality of Life; Physical Activity. 


\section{INTRODUÇÃO}

A hipertensão arterial resistente é caracterizada por altos níveis pressóricos, mesmo quando utilizado três classes de anti-hipertensivos, sendo um deles preferencialmente o diurético, ou quando são utilizados quatro ou mais anti-hipertensivos, independentemente dos valores pressóricos. ${ }^{1,2}$ Segundo o Posicionamento Brasileiro sobre Hipertensão Arterial Resistente, não há exata prevalência de indivíduos hipertensos resistentes no Brasil, uma vez que existem muitos casos de pseudo-resistência, como síndrome do jaleco branco e não adesão medicamentosa, mas há estimativa de que $12 \%$ a $15 \%$ dos hipertensos em geral apresentem resistência à terapia medicamentosa. ${ }^{3}$

De maneira geral, é bem consolidado que a fisiopatologia hipertensiva acarreta na diminuição da qualidade de vida relacionada à saúde. ${ }^{4-6}$ Por exemplo, Carvalho et $\mathrm{al}^{4}$ demonstraram que pacientes hipertensos, mesmo atendidos por equipe multiprofissional, apresentaram piora na qualidade de vida quando comparados aos seus pares normotensos. Esse achado foi diagnosticado pela diminuição da capacidade funcional, piora da saúde mental e estado geral da saúde reduzido destes pacientes. Nos pacientes com hipertensão arterial resistente o acometimento na qualidade de vida relacionada à saúde pode ser ainda maior. Estudo norte americano demonstrou que pessoas com diagnóstico de hipertensão resistente apresentam menores índices de qualidade de vida relacionado à saúde, quando comparados aos seus pares com diagnóstico de hipertensão arterial sistêmica. ${ }^{7}$

Por outro lado, na hipertensão arterial sistêmica é possível observar efeito positivo do estilo de vida fisicamente ativo na qualidade de vida relacionado à saúde. Arija et $a^{8}$ demonstraram que nove meses da prática de atividade física, compreendida em caminhar 120 minutos por semana, melhorou a qualidade de vida relacionada à saúde de pacientes com hipertensão arterial sistêmica, independente da mudança alimentar. Desta forma, é possível pensar que a prática regular de atividade física também poderá ter efeitos positivos na qualidade de vida relacionada à saúde de pessoas com hipertensão arterial resistente, fato desconhecido na literatura até o presente momento.

Com isso, o objetivo do presente estudo foi testar a hipótese de que, pacientes hipertensos resistentes fisicamente ativos apresentam melhor qualidade de vida relacionada à saúde quando comparados a indivíduos hipertensos resistentes sedentários.

\section{MATERIAL E MÉTODOS}

Estudo com delineamento transversal, realizado no Centro Hiperdia Minas, do Instituto Mineiro de Estudo Pesquisa em Nefrologia, localizado na cidade de Juiz de Fora/MG. Essa pesquisa foi aprovada pelo Comitê de Ética em Pesquisa com Seres Humanos do Hospital Universitário de Universidade Federal de Juiz de Fora sob o parecer número 766.317 .

O cálculo amostral foi realizado pelo software G*Power, versão 3.1.9.4 (Franz Faul, Universitat Kiel, Germany), no qual foi considerado poder de $78 \%$ e erro a de $5 \%$. De acordo com este cálculo, deveriam ser recrutados no mínimo 38 voluntários divididos em dois grupos. Para obtenção da amostra foi realizada busca ativa em 1043 prontuários, no Centro Hiperdia Minas. Foram incluídos indivíduos de ambos os gêneros, com idade entre 18 e 80 anos, não etilistas, não tabagistas e com índice de massa corpórea menor ou igual a $35 \mathrm{~kg} / \mathrm{m}^{2}$. Pacientes com histórico de acidente vascular encefálico e/ou infarto agudo do miocárdio foram excluídos. Após avaliação médica também foram excluídos os casos de pseudo-resistência. ${ }^{9}$ A amostra final contou com 38 voluntários hipertensos resistentes, sendo 19 fisicamente ativos (grupo Ativo) e 19 sedentários (grupo Sedentário).

Foi considerado fisicamente ativo o paciente que praticava regularmente exercício físico por pelo menos três vezes por semana, com duração mínima de duas horas semanais, por período superior a quatro meses anteriores à coleta de dados. Indivíduos do grupo sedentário se caracterizaram por não praticar exercício físico regularmente há, no mínimo, seis meses anteriores à coleta dos dados.

Após análise do prontuário, os participantes foram contatados e foi agendada visita na Unidade de Investigação Cardiovascular e Fisiologia do Exercício (InCFEx), do Hospital Universitário da Universidade Federal de Juiz de Fora, no período compreendido entre 13 e 18 horas. Nessa visita, a coleta de dados foi iniciada após o voluntário ler, concordar e assinar o termo de consentimento livre e esclarecido.

A qualidade de vida relacionada à saúde foi avaliada por meio do questionário SF-36, validado para a língua portuguesa/Brasil. ${ }^{10}$ GERD-HRQL (Gastroesophageal Reflux Disease - Health Related Quality of Life Esse instrumento é composto por 36 itens e divide-se em oito domínios: capacidade funcional, limitação por aspectos físicos, dor, estado geral de saúde, vitalidade, aspectos sociais, aspectos emocionais e saúde mental. Para sua avaliação é dado um escore para cada questão que depois são transformados numa escala de 0 a 100, onde zero é o pior nível e 100 é o melhor nível de qualidade de vida. ${ }^{11}$ Os voluntários responderam o questionário SF-36 por meio do autopreenchimento.

A estatura e massa corporal foram medidas pela balança e estadiômetro Líder ${ }^{\circledR}$ com precisão de $0,1 \mathrm{~kg}$. O índice de massa corpórea foi calculado utilizando a divisão do valor da massa corporal pelo quadrado da altura. ${ }^{12}$

As informações referentes às comorbidades associadas a hipertensão arterial resistente foram obtidas 
para cada participante via prontuário do Centro Hiperdia Minas.

Os valores foram expressos em média \pm desvio padrão. Para testar a normalidade dos dados foi realizado o Teste de Kolmogorov-Smirnov. Para testar a possível diferença de proporção da distribuição dos gêneros entre os grupos e relação de comorbidades associadas foi utilizado o teste exato de Fisher. Para a comparação dos escores de qualidade de vida relacionada à saúde entre os grupos foi realizado o teste $t$ de Student para amostras independentes. Foi considerado significativo nível de $\mathrm{p}<0,05$ e utilizado o programa STATISTICA, versão 8.0 (StatSoft, USA).

\section{RESULTADOS}

Os grupos Ativo e Sedentário foram semelhantes quanto a proporção dos gêneros, massa corporal, estatura, índice de massa corpórea e para o número de medicamentos anti-hipertensivos em vigência, como descrito na tabela 1 . Porém, os pacientes fisicamente ativos foram significativamente mais velhos quando comparados aos pacientes sedentários (tabela 1).

No que diz respeito à presença de comorbidades associadas à hipertensão, não houve diferença significativa entre os hipertensos resistentes fisicamente ativos e sedentários (tabela 2 ).

Os dados referentes à qualidade de vida relacionada à saúde dos grupos estão descritos na figura 1. O grupo Ativo apresentou escores significativamente superiores para os domínios capacidade funcional, dor, estado geral de saúde, vitalidade, limitação por aspectos físicos e saúde mental em comparação com o grupo Sedentário. Apesar disso, não foram observadas diferenças significativas entre os grupos estudados para os domínios aspectos sociais e limitação por aspectos emocionais.

Tabela 1: comparação das características demográficas e antropométricas entre os grupos Ativo e Sedentário.

\begin{tabular}{|c|c|c|c|}
\hline Variável & Grupo Ativo $(n=19)$ & Grupo Sedentário $(n=19)$ & $P$ \\
\hline Gênero (M/F) & $9 / 10$ & $5 / 14$ & 0,16 \\
\hline Idade (anos) & $64 \pm 7$ & $56 \pm 10$ & 0,01 \\
\hline Massa corporal $(\mathrm{kg})$ & $78 \pm 11$ & $74 \pm 11$ & 0,49 \\
\hline Estatura (m) & $1,62 \pm 0,08$ & $1,59 \pm 0,08$ & 0,27 \\
\hline $\begin{array}{l}\text { IMC }\left(\mathrm{kg} / \mathrm{m}^{2}\right) \\
\text { Anti-hipertensivos }\left(\mathrm{n}^{0}\right)\end{array}$ & $\begin{array}{c}30 \pm 4 \\
4(2)\end{array}$ & $\begin{array}{c}29 \pm 3 \\
4(2) \\
\end{array}$ & $\begin{array}{l}0,67 \\
0,44 \\
\end{array}$ \\
\hline
\end{tabular}

Tabela 2: Comparação das proporções de comorbidades a hipertenção arterial resistente entre os grupos Ativo e Sedentário.

\begin{tabular}{|c|c|c|c|}
\hline Comorbidades & $\begin{array}{l}\text { Grupo Ativo } \\
(n=19)\end{array}$ & $\begin{array}{c}\text { Grupo Sedentário } \\
\qquad(\mathrm{n}=19)\end{array}$ & $\mathbf{P}$ \\
\hline Diabetes Mellitus tipo 2 & 11 & 14 & 0,50 \\
\hline Dislipidemia & 17 & 13 & 0,23 \\
\hline Hipertrofia Ventricular Esquerda & 1 & 2 & 1,00 \\
\hline Hipotireoidismo & 2 & 0 & 0,49 \\
\hline Doença Renal Crônica & 3 & 0 & 0,23 \\
\hline Retinopatia Hipertensiva & 1 & 0 & 1,00 \\
\hline Pré-diabetes & 1 & 1 & 1,00 \\
\hline
\end{tabular}

Valores expressos como frequência absoluta de cada comorbidade por grupo, com análise do teste exato de Fisher. 

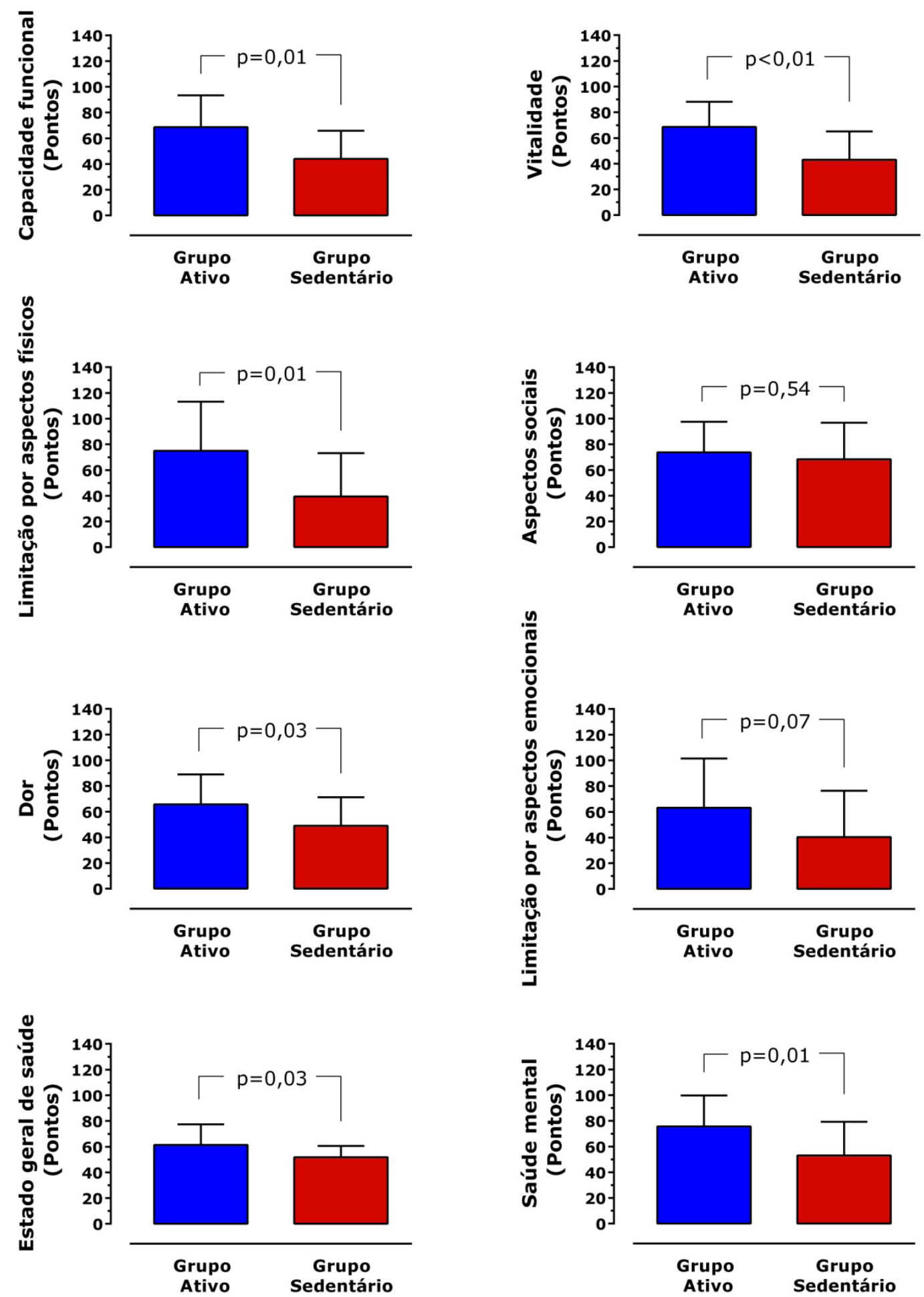

Figura 1: Comparação dos domínios de qualidade de vida relacionada saúde entre o Grupo Ativo e o Grupo Sedentário. Para todas as variáveis foi utilizado o teste t de Student para amostras independentes. 


\section{DISCUSSÃO}

Ao se tratar da fisiopatologia hipertensiva, os resultados do presente estudo estão coerentes com os da literatura. Avaliando mulheres com hipertensão arterial sistêmica, Póvoa et al $^{13}$ verificaram que oito semanas de treinamento físico aeróbio ou treinamento físico resistido foram suficientes para melhorar a qualidade de vida relacionada à saúde dessas pacientes, independentemente do tipo de treinamento físico empregado. Porém, o presente estudo parece ser o primeiro trabalho que investigou, em hipertensos resistentes, a melhora na qualidade de vida relacionada à saúde dependente da prática de atividade física.

Alguns fatores podem explicar, pelo menos em parte, a melhora da qualidade de vida relacionada à saúde provocada pela prática de atividade física nos domínios avaliados. ${ }^{14}$ Está bem documentado que pessoas fisicamente ativas, mesmo que não estejam engajadas em programa de treinamento físico sistematizado, possuem maior percentagem de massa muscular esquelética $e^{15}$ consequente, maior nível de força muscular que seus pares sedentários. ${ }^{15,16} \mathrm{Em}$ conjunto com a melhora da flexibilidade, ${ }^{17}$ esses fatores podem promover melhora na quantidade e na qualidade de execução das atividades físicas diárias após período de atividade física. ${ }^{18}$

Outro ponto interessante é que o exercício físico tem influência direta na saúde mental. Em estudo transversal, analisando dados de 1.237 .194 pessoas, com idade de 18 anos ou mais, foi observado que os indivíduos que se exercitavam fisicamente tiveram, aproximadamente, redução de $43 \%$ no número de dias ruins no último mês relacionados a saúde mental em comparação aos que não se exercitaram fisicamente nesse período.19 Esses dados reforçam os achados do presente estudo, onde foi observada melhora da saúde mental dos hipertensos resistentes com a prática de atividade física.

A prática regular de atividades físicas não interferiu nos domínios aspectos sociais e limitação por aspectos emocionais no presente estudo. Carris et $\mathrm{al}^{7}$ demonstraram que as comorbidades associadas podem afetar negativamente o aspecto emocional dos hipertensos resistentes. Apesar do presente estudo não ter demonstrado eficiência significativa nos hipertensos fisicamente ativos, foi observado que os grupos Ativo e Sedentário foram semelhantes para a presença das comorbidades cardiometabólicas associadas. Entretanto, o grupo Ativo apresentou melhor desempenho nesse domínio.

Os aspectos sociais podem ser afetados pela consciência da doença, pois muitas vezes se faz necessário mudar os hábitos alimentares e estilo de vida. De acordo com o estudo de Brito et $a^{20}$, essas mudanças podem implicar na ausência em reuniões sociais na tentativa de evitar o consumo de alimentos inadequados, levando o indivíduo a perdas nos relacionamentos sociais e momentos de lazer. No presente trabalho, não foi avaliado o critério conhecimento da doença e hábitos alimentares. De qualquer forma, atividade física em grupo é mais indicada para o desenvolvimento do aspecto social e somente três pacientes estavam envolvidos em atividade físicas coletivas. ${ }^{19}$

A diferença na idade poderia ser fator limitante da atual pesquisa. Porém, o grupo mais velho, que teria prejuízo adicional a qualidade de vida relacionada à saúde, ${ }^{21,22}$ foi o que apresentou os maiores índices. Isso demonstra que a prática regular de atividade física, de fato, pode interferir na melhora da qualidade de vida relacionada à saúde destas pessoas. Outro fator limitante é a presença de comorbidades associadas, não isolando a hipertensão resistente. Mas, vale ressaltar, que os grupos foram semelhantes quanto a distribuição das comorbidades, o que minimizou essa questão.

\section{CONCLUSÃO}

O presente estudo revelou que pacientes com hipertensão arterial resistente, quando fisicamente ativos, apresentam maior qualidade de vida relacionada à saúde, especificamente nos domínios capacidade funcional, dor, estado geral da saúde, vitalidade, limitação por aspectos físicos e saúde mental. Os domínios limitação por aspectos sociais e limitação por aspectos emocionais parecem não ser influenciados pelo estilo de vida fisicamente ativo. Desta forma, a prática regular de atividades físicas melhora a qualidade de vida relacionada à saúde desses pacientes.

\section{FINANCIAMENTO}

O presente trabalho foi realizado com apoio da Coordenação de Aperfeiçoamento de Pessoal de Nível Superior - Brasil (CAPES) - Código de Financiamento 001. E, apoio da Fundação de Amparo à Pesquisa do Estado de Minas Gerias (FAPEMIG).

\section{AGRADECIMENTO}

À Fundação Instituto Mineiro de Estudos e Pesquisas em Nefrologia e aos funcionários desta instituição por possibilitarem acesso aos prontuários médicos do serviço, bem como ao Hospital Universitário da UFJF e seus respectivos funcionários. E ainda, agradecemos a todos os pacientes que se colocaram à disposição para que este estudo pudesse ser realizado.

\section{REFERÊNCIAS}

1. Calhoun DA, Jones D, Textor S, Goff DC, Murphy TP, Toto RD et al. Resistant hypertension: diagnosis, evaluation, and 
treatment. Circulation. 2008; 117(25):e510-26. doi: 10.1161/ CIRCULATIONAHA.108.1891412.

2. Freitas IMG, Aleida LB, Pereira NP, Mira PAC, Paula RB, Martinez DG et al. Baroreflex gain and vasomotor sympathetic modulation in resistant hypertension. Clin Auton Res. 2017; 27(3):175-84.doi: 10.1007/s10286-017-0417-7

3. Departamento de Hipertensão Arterial da Sociedade Brasileira de Cardiologia. I Posicionamento Brasileiro Sobre Hipertensão Arterial Resistente. Arq Bras Cardiol. 2012; 99(1):576-85. doi: doi.org/10.1590/S0066-782X2012001000002

4. Carvalho MV, Siqueira LB, Sousa ALL, Jardim PCBV. The influence of hypertension on quality of life. Arq Bras Cardiol. 2013; 100(2):164-74. doi: 10.5935/abc.20130030

5. Cavalcante MA, Bombig MT, Luna Filho B, Carvalho AC, Paola $A A$, Póvoa R. Quality of life of hypertensive patients treated at an outpatient clinic. Arq Bras Cardiol. 2007; 89(4):245-50. doi: $10.1590 /$ s0066-782x2007001600006

6. Carvalho MA, Silva IB, Ramos SB, Coelho LF, Gonçalves ID, Figueiredo Neto JA. Quality of life of hypertensive patients and comparison of two instruments of HRQOL measure. Arq Bras Cardiol. 2012; 98(5):442-51. doi: 10.1590/s0066$782 \times 2012005000032$

7. Carris NW, Ghushchyan V, Libby AM, Smith SM. Healthrelated quality of life in persons with apparent treatmentresistant hypertension on at least four antihypertensives. J Hum Hypertens. 2016; 30(3):191-6. doi: 10.1038/jhh.2015.61

8. Arija V, Villalobos F, Pedret R, Vinuesa A, Jovani D, Pascual $G$ et al. Physical activity, cardiovascular health, quality of life and blood pressure control in hypertensive subjects: randomized clinical trial. Health Qual Life Outcomes. 2018; 16(1):184. doi: 10.1186/s12955-018-1008-6

9. Calhoun, D. A. Apparent and true resistant hypertension: why not the same? J Am Soc Hypertens. 2013; 7(6):509-11. doi: 10.1016/j.jash.2013.07.007

10. Pereira GI, Costa CD, Geocze L, Borim AA, Ciconelli RM, Camacho-Lobato L. Cross-cultural adaptation and validation for Portuguese (Brazil) of health related quality of life instruments specific for gastroesophageal reflux disease. Arq Gastroenterol. 2007; 44(2):168-77.

11. Saço LF, Paula OR, Migliorini GE, Pereira NP, Ferreira EL. Características e avaliação da qualidade de vida em um grupo de pacientes submetidos a tratamento quimioterápico. HU Rev. 2011; 37:95-102.

12. Associação Brasileira para o Estudo da Obesidade e da Síndrome Metabólica. Diretrizes Brasileiras de Obesidade 2016. 4. ed. São Paulo: ABESO; 2016.
13. Póvoa TIR et al. Treinamento aeróbio e resistido, qualidade de vida e capacidade funcional de hipertensas. Rev Bras Med Esporte. 2014; 20(1):36-41.

14. Silva RS, Silva I, Silva RA, Souza L, Tomasi E. Atividade física e qualidade de vida. Cien Saude Colet. 2010; 15:115-20.

15. Taylor AH, Cable NT, Faulkner G, Hillsdon M, Narici M, Van Der Bij AK. Physical activity and older adults: a review of health benefits and the effectiveness of interventions. J Sports Sci. 2004; 22(8):703-25. doi: 10.1080/02640410410001712421

16. Straight CR, Brady AO, Evans EM. Moderate-intensity physical activity is independently associated with lower-extremity muscle power in older women. Women Health. 2016; 56(8):871-84. do i: $10.1080 / 03630242.2016 .1141828$

17. Cayco CS, Labro AV, Gorgon EJR. Hold-relax and contractrelax stretching for hamstrings flexibility: a systematic review with meta-analysis. Phys Ther Sport. 2019; 35:42-55. doi: 10.1016/j.ptsp.2018.11.001

18. Martins AVV, Neto JAC, Ferreira RE, Souza DZO, Pereira FPS, Gasparoni JM. As atitudes e o conhecimento sobre práticas de vida saudáveis de uma amostra da população de Juiz de Fora. HU Rev. 2017; 43(1):51-60.

19. Chekroud SR, Gueorguieva R, Zheutlin AB, Paulus M, Krumholz HM, Krystal JH et al. Association between physical exercise and mental health in 1.2 million individuals in the USA between 2011 and 2015: a cross-sectional study. The Lancet Psychiatry. 2018; 5(9):739-46. doi: 10.1016/S22150366(18)30227-X

20. Brito DMS, Araújo TL, Galvão MTG, Moreira TMM, Lopes MV de O. Qualidade de vida e percepção da doença entre portadores de hipertensão arterial. Cad Saude Publica. 2008; 24(4):93340. doi: $10.1590 /$ s0102-311×2008000400025

21. Guedes DV, Silva KCA, Banhato EFC, Mota MMPE. Fatores associados à capacidade funcional de idosos da comunidade. HU Rev. 2007; 33(4):105-11.

22. Teixeira JS, Ferreira MEC. Metanálise de pesquisas sobre qualidade de vida, saúde e bem-estar subjetivo no envelhecimento. HU Rev. 2015; 41(1e2):41-53. 